\title{
Article \\ Changes in Peripapillary and Macular Vessel Densities and Their Relationship with Visual Field Progression after Trabeculectomy
}

\author{
Jooyoung Yoon, Kyung Rim Sung * and Joong Won Shin
}

Citation: Yoon, J.; Sung, K.R.; Shin, J.W. Changes in Peripapillary and Macular Vessel Densities and Their Relationship with Visual Field Progression after Trabeculectomy. J. Clin. Med. 2021, 10, 5862. https:// doi.org/10.3390/jcm10245862

Academic Editors: Vito Romano, Yalin Zheng and Mariantonia Ferrara

Received: 8 November 2021

Accepted: 13 December 2021

Published: 14 December 2021

Publisher's Note: MDPI stays neutral with regard to jurisdictional claims in published maps and institutional affiliations.

Copyright: (c) 2021 by the authors. Licensee MDPI, Basel, Switzerland. This article is an open access article distributed under the terms and conditions of the Creative Commons Attribution (CC BY) license (https:/ / creativecommons.org/licenses/by/ $4.0 /)$.
Department of Ophthalmology, College of Medicine, University of Ulsan, Asan Medical Center, Seoul 05505, Korea; cec1204@naver.com (J.Y.); sideral@hanmail.net (J.W.S.)

* Correspondence: sungeye@gmail.com; Tel.: +82-2-3010-3680; Fax: +82-2-470-6440
Abstract: The aim of this study was to determine the factors associated with visual field (VF) deterioration after trabeculectomy, including the peripapillary vessel density ( $p V D$ ) and macular vessel density (mVD) changes assessed by optical coherence tomography angiography (OCT-A). Primary open-angle glaucoma patients with more than two years of follow-up after trabeculectomy were included. pVD was calculated in a region defined as a $750 \mu \mathrm{m}$-wide elliptical annulus extending from the optic disc boundary. mVD was calculated in the parafoveal (1-3 mm) and perifoveal (3-6 $\mathrm{mm}$ ) regions. VF deterioration was defined as the rate of mean deviation (MD) worse than $-1.5 \mathrm{~dB}$ /year. The change rates of $\mathrm{pVD}$ and $\mathrm{mVD}$ were compared between the deteriorated VF and non-deteriorated VF groups. The factors associated with the rate of MD were determined by linear regression analyses. VF deterioration was noted in $14(21.5 \%)$ of the 65 eyes that underwent trabeculectomy. The $\mathrm{pVD}(-2.26 \pm 2.67$ vs. $-0.02 \pm 1.74 \%$ /year, $p \leq 0.001)$ reduction rate was significantly greater in the deteriorated VF group than in the non-deteriorated VF group, while that of parafoveal $(p=0.267)$ and perifoveal $(p=0.350)$ VD did not show a significant difference. The linear regression analysis showed that the postoperative MD reduction rate was significantly associated with the rate of $\mathrm{pVD}$ reduction $(p=0.016)$, while other clinical parameters and preoperative vascular parameters did not show any association. Eyes with greater loss of peripapillary retinal circulation after trabeculectomy tended to exhibit VF deterioration. The assessment of peripapillary vascular status can be an adjunctive strategy to predict visual function after trabeculectomy.

Keywords: primary open-angle glaucoma; optical coherence tomography angiography; vessel density; visual field; progression

\section{Introduction}

Glaucoma causes progressive structural abnormalities in the optic nerve head $(\mathrm{ONH})$ and the loss of visual function [1]. The mainstay of glaucoma treatment is slowing down the disease progression by preventing further $\mathrm{ONH}$ damage. The lowering of intraocular pressure (IOP) is the only proven way to slow down the disease progression [2,3]. Elevated IOP is a well-known risk factor for glaucoma [4-7]; however, several vascular factors also play a role in the pathophysiology of glaucoma development and progression [8]. Previous studies have evaluated the changes of the ocular hemodynamic in the ophthalmic artery, $\mathrm{ONH}$, and retinal vasculature $[9,10]$ after medical or surgical IOP reduction.

OCT angiography (OCT-A) is a non-invasive technique that can provide quantitative and reproducible vascular information of the $\mathrm{ONH}$ and retina [11]. OCT-A allows the precise visualization of the retinal capillary network [12] layer by layer, and a reduction of the vessel density (VD) assessed by OCT-A showed a correlation with the structural and functional parameters in glaucoma patients [13-15].

Increased or stable microcirculation after the surgical lowering of IOP has been reported [16-20]. However, research outcomes evaluating the clinical implication of postop- 
erative microvascular changes in association with the visual prognosis are limited. Hence, we aimed to evaluate the longitudinal peripapillary and macular microcirculation changes after the surgical IOP reduction and their association with the postoperative visual field (VF) changes.

\section{Materials and Methods}

In this retrospective observational study, we recruited primary open-angle glaucoma (POAG) patients who underwent trabeculectomy by a single surgeon (KRS) at the glaucoma clinic of Asan Medical Center (Seoul, Korea) between November 2016 and January 2019. All the study procedures were performed in accordance with the principles of the Declaration of Helsinki. The Institutional Review Board of Asan Medical Center approved this study. The requirement for written informed consent was waived due to the retrospective design.

\subsection{Participants}

At the baseline examination, all the participants underwent complete ophthalmologic examinations; best-corrected visual acuity (BCVA), refractometry, slit-lamp biomicroscopy, Goldmann applanation tonometry, gonioscopy, stereoscopic optic disc/retinal nerve fiber layer (RNFL) photography, ultrasound pachymetry, standard automated perimetry (Humphrey Field analyzer with Swedish Interactive Threshold Algorithm standard 24-2 test; Carl Zeiss Meditec, Dublin, CA, USA), and OCT-A (AngioVue, Optovue Inc., Fremont, CA, USA).

The inclusion criteria of this study were as follows: patients diagnosed with POAG, with BCVA of logMAR +0.30 (Snellen 20/40) or better, a spherical refraction of -8.0 to +3.0 diopters (D), a cylinder correction within $\pm 3 \mathrm{D}$, and clear ocular media. POAG was defined as having an open angle on gonioscopy, RNFL defects, or glaucomatous optic disc changes (neuroretinal rim thinning, disc excavation, or disc hemorrhage), and corresponding VF defects. Participants with any ophthalmic or neurological disease other than glaucoma that can affect $\mathrm{ONH}$ were excluded. If both eyes met the inclusion criteria, one eye was selected at random. The participants were followed-up for $\geq 2$ years after the trabeculectomy.

Trabeculectomy was performed in patients with progressive glaucomatous changes that could not be controlled with maximum tolerated medical therapy (MTMT) and in those with an elevated IOP that could cause additional ONH damage. A single experienced glaucoma specialist (KRS) performed all surgical interventions. All glaucoma medications were continued up to the time of the surgery. Eyes with persistent hypotony maculopathy after trabeculectomy or eyes with other macular abnormalities, such as an epiretinal membrane or age-related macular degeneration, were excluded from the study. Patients who received other intraocular surgery during the 2-year follow-up period were also excluded from the study.

\subsection{Trabeculectomy}

Trabeculectomy was performed by a single surgeon (KRS). A 6- to 7-mm horizontal incision was made in the superior area, and the conjunctiva and Tenon's capsule were carefully dissected for preparation of the fornix-based conjunctival flap. A limbus-based half-thickness scleral flap $(2.5 \times 2 \mathrm{~mm})$ was then prepared. $0.2 \%$ mitomycin C-soaked sponge was applied under the sub-Tenon space for $2 \mathrm{~min}$ and copious irrigation with balanced salt solution (BSS) was performed in order to wash out the mitomycin $\mathrm{C}$. The sclerectomy was made with the Kelly Descemet punch under the partial scleral flap, and the peripheral iridectomy was performed through the sclerectomy site. The scleral flap was closed with a single 9-0 nylon suture. The conjunctiva and Tenon's capsule are secured with a 8-0 vicryl interrupted suture followed by running sutures. Bleb elevation and integrity of the conjunctival closure were checked. Topical corticosteroid (1.0\% prednisolone), cycloplegics, and an antibiotic ( $0.5 \%$ moxifloxacin) were prescribed for approximately 1 month postoperatively, depending on the eye condition. 


\subsection{VF Assessment}

Only the reliable VF test results were included in the analysis. A reliable VF test result was defined by the presence of false-positive errors $<15 \%$, false-negative errors $<15 \%$, and fixation loss $<20 \%$. A glaucomatous VF defect was defined as the presence of a cluster of three or more non-edge contiguous points on a pattern deviation plot with a $p$-value $<5 \%$ (one of which had a $p$-value $<1 \%$ ), confirmed by at least two consecutive examinations; pattern standard deviation with a $p$-value $<5 \%$; or glaucoma hemifield test result outside normal limits. The VF was assessed before the surgery, and 6 months, 1 year, 1.5 years, and 2 years postoperatively. VF progression was defined as the rate of mean deviation (MD) worse than $-1.5 \mathrm{~dB}$ annually [21]. At least five qualified VF examinations were required to be included.

\subsection{OCT-A Imaging}

The AngioVue OCT-A imaging system enables non-invasive visualization of the ophthalmic microvasculature. The dynamic motion of the moving particles, such as red blood cells, was captured using this system, with a split-spectrum amplitude-decorrelation angiography algorithm used to identify the perfused vessels. In this study, the peripapillary vasculature was measured in a $4.5 \times 4.5 \mathrm{~mm}$ region centered on the optic disc, and within a slab from the internal limiting membrane to the posterior border of the RNFL. The peripapillary VD (pVD) was calculated in a region defined as a $750 \mu \mathrm{m}$-wide elliptical annulus extending from the optic disc boundary. The macular vasculature was measured in a $6.0 \times 6.0 \mathrm{~mm}$ region centered on the fovea, and within a slab from the internal limiting membrane to the posterior border of the inner plexiform layer. The macular VD was calculated in the parafoveal and perifoveal regions, defined as concentric circular areas with an inner and outer diameter of $1 \mathrm{~mm}$ and $3 \mathrm{~mm}$, and $3 \mathrm{~mm}$ and $6 \mathrm{~mm}$, respectively. All the scans were evaluated for quality. The reasons for exclusion were poor image quality, defined as the signal strength index $<48$; poor clarity; localized weak signals caused by artifacts, such as floaters; residual motion artifacts visible as irregular vessel patterns or disc boundaries on the en face angiogram; or segmentation failure. At least five qualified OCT-A examinations were required to be included. The circumpapillary retinal nerve fiber layer (RNFL) thickness and ganglion cell complex (GCC) thickness were also assessed using the same device.

\subsection{Statistical Analysis}

The normality was tested using the Kolmogorov-Smirnov test for all continuous variables. The baseline clinical characteristics were compared between the eyes with relatively stable VF and those with deteriorated VF after trabeculectomy using either an independent t-test or Mann-Whitney test as appropriate. A linear mixed model was performed to determine the postoperative change rate of IOP, and the OCT-A driven vascular and structural parameters. Repeated measures ANOVA was used to compare the postoperative changes of each parameter. Univariate and multivariate linear regression analyses, including the OCT-A driven vascular parameters, were used to determine the factors associated with the VF MD change rate after the trabeculectomy. A $p$-value $<0.1$ in the univariate analysis was included in the multivariate analysis. The statistical analysis was performed using the SPSS software, version 20 (IBM Corp., Armonk, NY, USA). $p$-value $\leq 0.05$ was considered statistically significant.

\section{Results}

Of the 81 eyes of 81 patients initially enrolled, 16 eyes were excluded due to the poor quality, poor clarity, and segmentation failure of the OCT-A image. Sixty-five eyes of 65 patients ( 38 male, 27 female) with successful trabeculectomy and qualified visual field tests were included in the final analysis. The baseline characteristics are described in Table 1. Postoperative VF deterioration was observed in 14 eyes (21.5\%) when assessed for two years postoperatively. The preoperative clinical characteristics, including age, VF 
MD, and IOP did not differ between the two groups. VD parameters, RNFL, and GCC thickness, which were assessed before surgery, also did not differ between the groups. No significant postoperative complications, such as prolonged hypotony and inadequate IOP control, were observed in both groups. Topical medications were prescribed based on the patients' postoperative condition. $1.59 \pm 1.03$ topical medications were used in the total population after the surgery, while $1.57 \pm 1.02$ in the progression group, and $1.59 \pm 1.04$ in the non-progression group were used, respectively. The difference in the number of postoperative topical medications between the progression and non-progression groups was not statistically significant ( $p=0.957)$.

Table 1. Baseline demographics and the clinical characteristics of the VF progression and non-progression group.

\begin{tabular}{|c|c|c|c|c|}
\hline Variables & $\begin{array}{c}\text { Total } \\
(n=65)\end{array}$ & $\begin{array}{l}\text { Progression } \\
\quad(n=14)\end{array}$ & $\begin{array}{c}\text { Non-Progression } \\
\quad(n=51)\end{array}$ & $p$-Value * \\
\hline Age (years) & $54.8 \pm 13.3$ & $55.7 \pm 13.5$ & $54.6 \pm 13.4$ & 0.778 \\
\hline Sex, male/female & $38 / 27$ & $8 / 6$ & $30 / 21$ & 0.911 \\
\hline Topical medications, $n$ & $2.8 \pm 0.6$ & $2.6 \pm 0.5$ & $2.8 \pm 0.6$ & 0.322 \\
\hline Self-reported history of HTN, $n(\%)$ & $20(30.8 \%)$ & $4(28.6 \%)$ & $16(31.4 \%)$ & 0.842 \\
\hline Self-reported history of DM, $n(\%)$ & $8(12.3 \%)$ & $2(14.3 \%)$ & $6(11.8 \%)$ & 0.801 \\
\hline VF MD $(\mathrm{dB})$ & $-16.6 \pm 7.9$ & $-13.8 \pm 6.7$ & $-17.4 \pm 8.1$ & 0.137 \\
\hline IOP (mmHg) & $19.9 \pm 8.4$ & $20.5 \pm 7.9$ & $20.0 \pm 9.2$ & 0.860 \\
\hline SE (D) & $-2.3 \pm 3.1$ & $-3.0 \pm 3.6$ & $-2.1 \pm 2.9$ & 0.301 \\
\hline Axial length (mm) & $24.9 \pm 1.8$ & $25.1 \pm 1.8$ & $24.9 \pm 1.7$ & 0.675 \\
\hline Central corneal thickness $(\mu \mathrm{m})$ & $530.1 \pm 45.5$ & $513.1 \pm 51.5$ & $533.8 \pm 43.8$ & 0.195 \\
\hline Peripapillary VD (\%) & $34.75 \pm 5.85$ & $34.25 \pm 5.55$ & $34.86 \pm 5.98$ & 0.780 \\
\hline Foveal VD (\%) & $15.99 \pm 6.46$ & $15.45 \pm 5.37$ & $16.14 \pm 6.77$ & 0.728 \\
\hline Parafoveal VD (\%) & $41.73 \pm 4.99$ & $39.77 \pm 4.78$ & $42.27 \pm 4.96$ & 0.098 \\
\hline Perifoveal VD (\%) & $37.75 \pm 3.80$ & $36.25 \pm 3.53$ & $38.16 \pm 3.80$ & 0.096 \\
\hline RNFL thickness ( $\mu \mathrm{m})$ & $65.92 \pm 8.83$ & $66.23 \pm 6.35$ & $65.84 \pm 9.41$ & 0.889 \\
\hline GCC thickness $(\mu \mathrm{m})$ & $69.59 \pm 7.48$ & $67.54 \pm 5.22$ & $70.12 \pm 7.92$ & 0.271 \\
\hline
\end{tabular}

Independent $t$-test for numerical variables; Mann-Whitney test for non-numerical variables. ${ }^{*} p \leq 0.05$ was considered statistically significant. Abbreviations: HTN, Hypertension; DM, Diabetes mellitus; VF MD, Visual field mean deviation; SE, Spherical equivalence; VD, vessel density; RNFL, Retinal nerve fiber layer; GCC, Ganglion cell complex.

The postoperative change rate of clinical parameters is described in Tables 2 and 3 , and Figure 1. The VF MD rate of the deteriorated VF group was $-2.46 \pm 0.77 \mathrm{~dB} /$ year, while that of the non-progression VF group was $0.06 \pm 0.89 \mathrm{~dB} /$ year $(p<0.001)$. The rate of $\mathrm{pVD}(p \leq 0.001)$ and RNFL thickness $(p=0.039)$ differed between the two groups, while foveal VD $(p=0.054)$ showed marginal difference. The parafoveal VD $(p=0.267)$ and perifoveal VD $(p=0.350)$ did not show significant difference. The rate of IOP $(p=0.672)$ and other structural parameters, such as GCC $(p=0.198)$ thickness, did not differ between the groups.

The baseline clinical characteristics and postoperative change rates of each OCT-A parameter were analyzed using univariate and multivariate linear regression analyses to determine the factors associated with the VF MD change rate after trabeculectomy (Table 4). None of the preoperative parameters showed an association with the postoperative VF MD rate. However, the rate of $\mathrm{pVD}(p=0.006)$ and foveal VD $(p=0.057)$ in the univariate analysis showed a possible association with the postoperative VF MD rate; additionally, the multivariate analysis revealed that only the postoperative reduction rate of $\mathrm{pVD}$ showed a correlation $(p=0.016)$.

A representative case example is shown in Figure 2. A 43-year-old woman with POAG exhibited a gradual loss of peripapillary microcirculation after trabeculectomy along with progressive glaucomatous VF progression (a). A 25-year-old woman with POAG showed stable peripapillary retinal microcirculation and VF after trabeculectomy when assessed for two years with a similar level of preoperative VF MD and IOP reduction after trabeculectomy $(b)$. 
Table 2. Comparison of the postoperative change rate of the parameters between the VF progression and non-progression group.

\begin{tabular}{ccccc}
\hline $\begin{array}{c}\text { Variables, } \\
\text { Mean } \pm \text { SD [95\% CI] }\end{array}$ & $\begin{array}{c}\text { Total } \\
(\boldsymbol{n}=\mathbf{6 5})\end{array}$ & $\begin{array}{c}\text { Progression } \\
(\boldsymbol{n}=\mathbf{1 4})\end{array}$ & $\begin{array}{c}\text { Non-Progression } \\
(\boldsymbol{n}=\mathbf{5 1})\end{array}$ & $\boldsymbol{p}$-Value * \\
\hline VF MD change rate & $-0.49 \pm 1.35$ & $-2.46 \pm 0.77$ & $0.06 \pm 0.89$ & $<0.001^{*}$ \\
(dB/year) & $(-0.82,-0.15)$ & $(-2.91,-2.01)$ & $(-0.19,0.30)$ & \\
IOP reduction rate & $-3.43 \pm 4.56$ & $-3.89 \pm 4.13$ & $-3.30 \pm 4.70$ & 0.672 \\
$\quad$ (mmHg/year) & $(-4.56,-2.30)$ & $(-6.28,-1.51)$ & $(-4.63,-1.98)$ & \\
Peripapillary VD & $-0.50 \pm 2.17$ & $-2.26 \pm 2.67$ & $-0.02 \pm 1.74$ & $<0.001^{*}$ \\
change rate (\%/year) & $(-1.04,0.04)$ & $(-3.81,-0.72)$ & $(-0.51,0.47)$ & \\
Foveal VD change rate & $-0.28 \pm 3.55$ & $-1.62 \pm 2.52$ & $0.09 \pm 3.73$ & 0.054 \\
$\quad(\% /$ year) & $(-1.16,0.60)$ & $(-3.08,-0.17)$ & $(-0.96,1.13)$ & \\
Parafoveal VD change & $-0.80 \pm 2.97$ & $-1.59 \pm 2.71$ & $-0.59 \pm 3.03$ & 0.267 \\
rate (\%/year) & $(-1.54,-0.07)$ & $(-3.15,-0.02)$ & $(-1.43,0.26)$ & \\
Perifoveal VD change & $-0.72 \pm 1.97$ & $-1.16 \pm 1.55$ & $-0.60 \pm 2.06$ & 0.350 \\
rate $(\% /$ year) & $(-1.21,-0.23)$ & $(-2.06,-0.27)$ & $(-1.18,-0.02)$ & \\
RNFL thickness change & $-0.70 \pm 3.82$ & $-2.64 \pm 3.80$ & $-0.20 \pm 3.70$ & $0.039 *$ \\
rate $(\mu \mathrm{m} /$ year) & $(-1.66,0.26)$ & $(-4.93,-0.34)$ & $(-1.25,0.85)$ & \\
GCC thickness change & $0.59 \pm 3.51$ & $1.72 \pm 3.52$ & $0.31 \pm 3.49$ & 0.198 \\
rate $(\mu \mathrm{m} /$ year) & $(-0.31,1.47)$ & $(-0.41,3.85)$ & $(-0.67,1.29)$ &
\end{tabular}

Independent $t$-test. ${ }^{*} p \leq 0.05$ was considered statistically significant. Abbreviations: VF MD, Visual field mean deviation; IOP, intraocular pressure; VD, vessel density; RNFL, Retinal nerve fiber layer; GCC, Ganglion cell complex.

Table 3. Comparison of the postoperative changes of VF MD, IOP and peripapillary VD between the VF progression and non-progression group.

\begin{tabular}{|c|c|c|c|c|c|}
\hline \multicolumn{2}{|c|}{$\begin{array}{c}\text { Variables, } \\
\text { Mean } \pm \text { SD }\end{array}$} & $\begin{array}{c}\text { Total } \\
(n=65)\end{array}$ & $\begin{array}{l}\text { Progression } \\
(n=14)\end{array}$ & $\begin{array}{c}\text { Non-Progression } \\
\quad(n=51)\end{array}$ & $p$-Value * \\
\hline \multirow{5}{*}{ VF MD (dB) } & Pre-op & $-16.62 \pm 7.92$ & $-15.32 \pm 5.91$ & $-17.19 \pm 8.01$ & \multirow{5}{*}{$<0.001^{*}$} \\
\hline & Post-op 0.5yr & $-16.88 \pm 8.01$ & $-17.49 \pm 7.23$ & $-16.47 \pm 8.27$ & \\
\hline & Post-op 1yr & $-17.01 \pm 7.81$ & $-18.88 \pm 5.88$ & $-16.39 \pm 8.06$ & \\
\hline & Post-op $1.5 y r$ & $-16.98 \pm 7.80$ & $-19.43 \pm 6.75$ & $-16.44 \pm 8.05$ & \\
\hline & Post-op 2yr & $-17.36 \pm 8.16$ & $-20.07 \pm 6.55$ & $-16.65 \pm 8.10$ & \\
\hline \multirow{5}{*}{$\mathrm{IOP}(\mathrm{mmHg})$} & Pre-op & $19.85 \pm 8.42$ & $20.50 \pm 7.92$ & $19.67 \pm 8.62$ & \multirow{5}{*}{0.789} \\
\hline & Post-op 0.5yr & $11.55 \pm 2.92$ & $11.57 \pm 2.53$ & $11.55 \pm 3.04$ & \\
\hline & Post-op 1yr & $12.42 \pm 2.97$ & $12.71 \pm 2.89$ & $12.33 \pm 3.01$ & \\
\hline & Post-op $1.5 y r$ & $12.78 \pm 2.92$ & $12.57 \pm 2.82$ & $12.84 \pm 2.98$ & \\
\hline & Post-op 2yr & $12.98 \pm 2.87$ & $12.71 \pm 2.52$ & $13.06 \pm 2.98$ & \\
\hline \multirow{5}{*}{$\begin{array}{l}\text { Peripapillary } \\
\text { VD }(\%)\end{array}$} & Pre-op & $34.75 \pm 5.85$ & $34.36 \pm 5.55$ & $34.86 \pm 5.98$ & \multirow{5}{*}{$0.001 *$} \\
\hline & Post-op 0.5yr & $33.92 \pm 6.33$ & $31.13 \pm 4.93$ & $34.62 \pm 6.51$ & \\
\hline & Post-op 1yr & $35.79 \pm 6.18$ & $30.42 \pm 3.89$ & $36.24 \pm 6.50$ & \\
\hline & Post-op $1.5 y r$ & $34.54 \pm 6.62$ & $31.18 \pm 3.20$ & $35.45 \pm 6.96$ & \\
\hline & Post-op 2yr & $32.27 \pm 4.22$ & $30.28 \pm 3.01$ & $32.47 \pm 4.35$ & \\
\hline
\end{tabular}

Repeated measures ANOVA * $p \leq 0.05$ was considered statistically significant. Abbreviations: VF MD, Visual field mean deviation; IOP, intraocular pressure; VD, vessel density. 

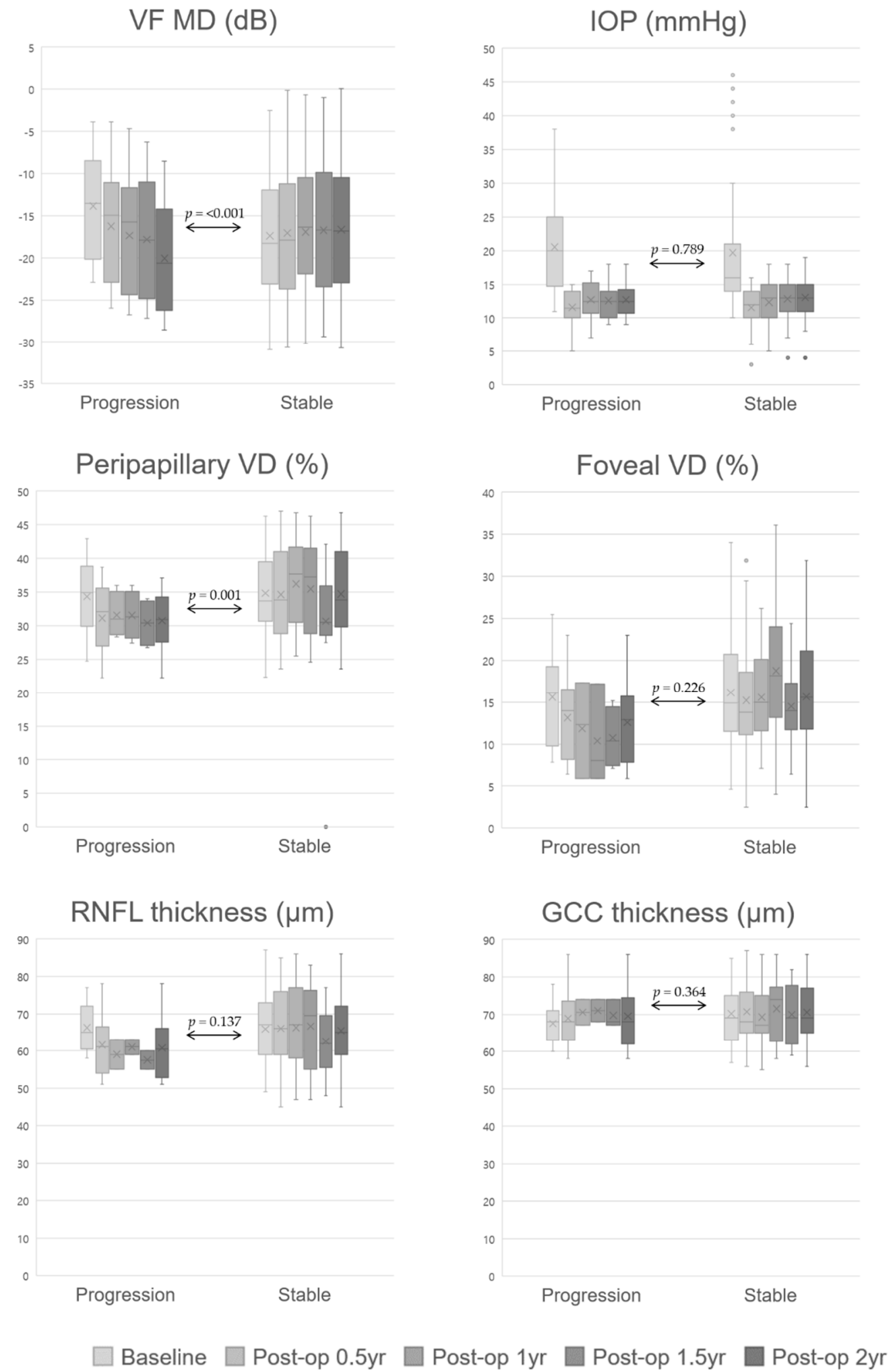

Figure 1. Comparison of postoperative parameter changes between the VF progression and non-progression group. Repeated measures ANOVA was used to compare the post-operative changes of each parameter, and the $p$-values of repeated measures ANOVA were presented. X: average value, horizontal line: median value, dots: outliers. Abbreviations: GCC, ganglion cell complex; IOP, intraocular pressure; RNFL, retinal nerve fiber layer; VD, vessel density; VF MD, visual field mean deviation. 
Table 4. Univariable and multivariable linear regression analyses to determine the factors associated with the visual field change rate after trabeculectomy.

\begin{tabular}{|c|c|c|c|c|}
\hline \multirow[t]{2}{*}{ Variables } & \multicolumn{2}{|c|}{ Univariable } & \multicolumn{2}{|c|}{$\begin{array}{c}\text { Multivariable } \\
(p<0.1 \text { in Univariable })\end{array}$} \\
\hline & $\mathrm{B} \pm \mathrm{SD}$ & $p$-Value * & $\mathbf{B} \pm \mathbf{S D}$ & $p$-Value * \\
\hline Age (years) & $0.003 \pm 0.013$ & 0.814 & & \\
\hline SE (D) & $0.018 \pm 0.055$ & 0.739 & & \\
\hline Central corneal thickness $(\mu \mathrm{m})$ & $0.005 \pm 0.004$ & 0.135 & & \\
\hline Baseline IOP (mmHg) & $-0.001 \pm 0.019$ & 0.961 & & \\
\hline Baseline VF MD (dB) & $-0.034 \pm 0.021$ & 0.112 & & \\
\hline Baseline peripapillary VD (\%) & $-0.013 \pm 0.029$ & 0.648 & & \\
\hline Baseline foveal VD (\%) & $-0.024 \pm 0.026$ & 0.369 & & \\
\hline Baseline parafoveal VD (\%) & $0.009 \pm 0.034$ & 0.800 & & \\
\hline Baseline perifoveal VD (\%) & $0.024 \pm 0.045$ & 0.586 & & \\
\hline Baseline RNFL thickness ( $\mu \mathrm{m})$ & $-0.013 \pm 0.019$ & 0.499 & & \\
\hline Baseline GCC thickness ( $\mu \mathrm{m})$ & $0.022 \pm 0.023$ & 0.341 & & \\
\hline Postoperative IOP reduction rate (mmHg/year) & $0.011 \pm 0.037$ & 0.771 & & \\
\hline Postoperative peripapillary VD change rate (\%/year) & $0.209 \pm 0.074$ & 0.006 & $0.186 \pm 0.075$ & 0.016 \\
\hline Postoperative foveal VD change rate $(\% /$ year $)$ & $0.090 \pm 0.046$ & 0.057 & $0.065 \pm 0.046$ & 0.160 \\
\hline Postoperative parafoveal VD change rate (\%/year) & $0.078 \pm 0.056$ & 0.175 & & \\
\hline Postoperative perifoveal VD change rate (\%/year) & $0.059 \pm 0.086$ & 0.496 & & \\
\hline RNFL thickness change rate ( $\mu \mathrm{m} /$ year $)$ & $0.075 \pm 0.044$ & 0.098 & & \\
\hline GCC thickness change rate ( $\mu \mathrm{m} /$ year $)$ & $-0.047 \pm 0.048$ & 0.335 & & \\
\hline
\end{tabular}

${ }^{*} p \leq 0.05$ was considered statistically significant. Abbreviations: SD, standard deviation; VF MD, visual field mean deviation; IOP, intraocular pressure; SE, spherical equivalence; VD, vessel density; RNFL, retinal nerve fiber layer; GCC, ganglion cell complex.
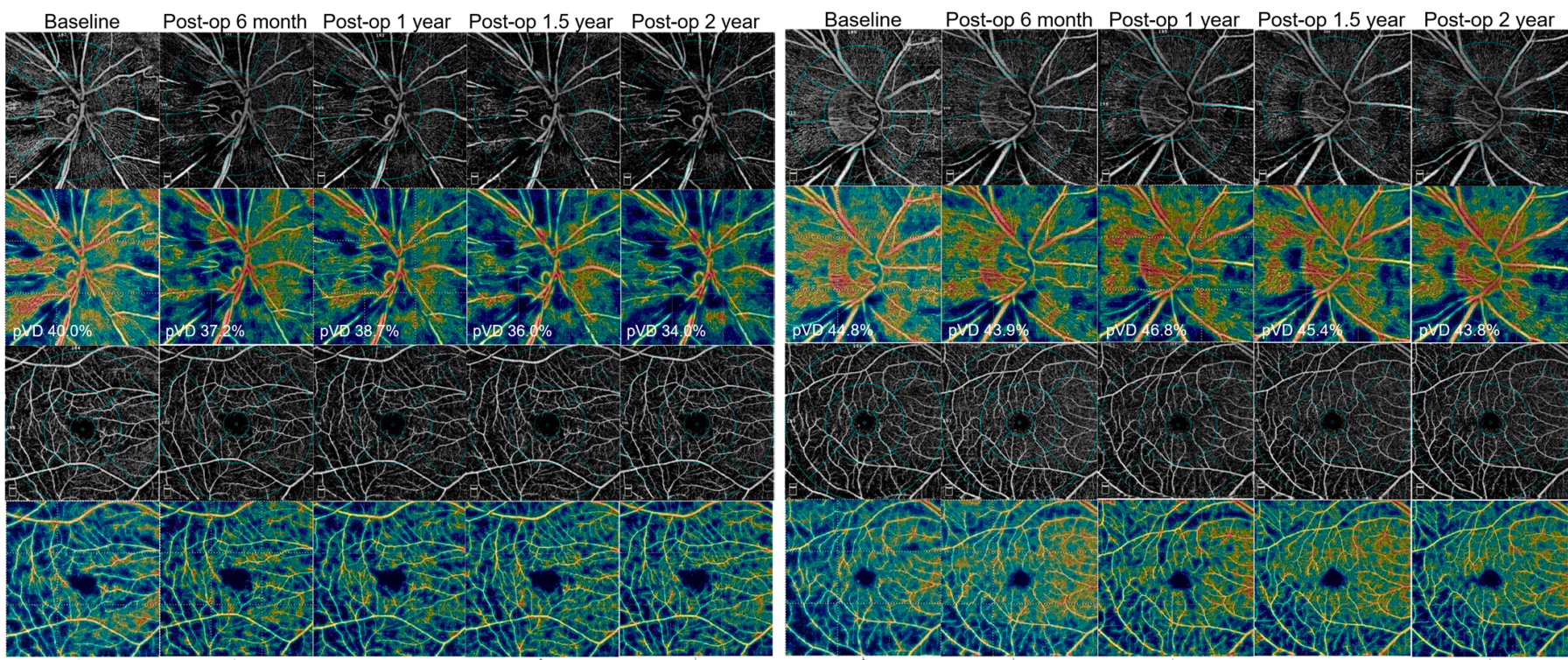

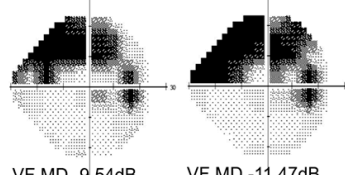

VF MD $-9.54 \mathrm{~dB}$

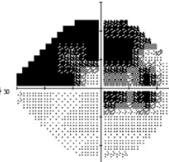

VF MD $-13.48 \mathrm{~dB}$

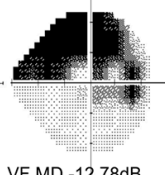

VF MD -12.78dB

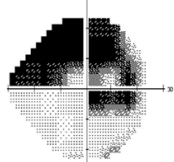

VF MD $-14.48 \mathrm{~dB}$

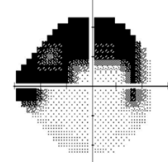

VF MD - $13.11 \mathrm{~dB}$

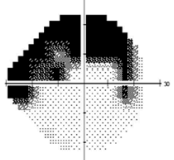

VF MD $-12.73 \mathrm{~dB}$

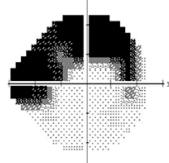

VF MD - $13.74 \mathrm{~dB}$

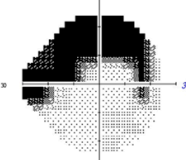

VF MD -13.93dB

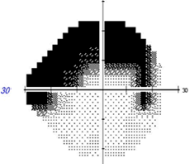

VF MD $-13.97 d B$

(a)

(b)

Figure 2. (a) A 43-year-old woman with POAG exhibited a gradual loss of peripapillary microcirculation after trabeculectomy along with progressive glaucomatous VF progression. (b) A 25-year-old woman with POAG showed stable peripapillary retinal microcirculation and VF after trabeculectomy when assessed for 2 years with a similar level of preoperative VF MD and IOP reduction after trabeculectomy. Abbreviations: POAG, primary open angle glaucoma; VF, visual field; IOP, intraocular pressure. 


\section{Discussion}

Our study demonstrated a two-year VF change after trabeculectomy in POAG eyes and the factors associated with the postoperative VF deterioration. The deteriorated VF group showed a faster rate of $\mathrm{pVD}$ than the non-deteriorated VF group. Furthermore, the rate of $\mathrm{pVD}$ was the only factor associated with that of VF MD. The baseline clinical characteristics or the change rates of other structural parameters, or macular area VD were not relevant to postoperative VF changes.

Trabeculectomy is the most commonly performed glaucoma surgery in patients with inadequate IOP control or progression of glaucoma despite MTMT. Trabeculectomy can slow the rate of glaucomatous deterioration; however, it does not completely stop the disease progression in the long term, and some studies reported the continuous deterioration of VF despite successful IOP control after trabeculectomy [22-24]. Hence, factors other than IOP should be sought and considered for the care of glaucoma patients who have undergone trabeculectomy. This study demonstrated the association between the postoperative $\mathrm{PVD}$ change and VF MD rates, which indicates that peripapillary retinal circulation change could provide insight into postoperative VF change in glaucoma patients.

Studies $[16-20,25]$ have evaluated the change of peripapillary and retinal microvasculature following a large amount of IOP reduction in glaucoma patients. Some studies $[17,18,20]$ reported limited or no significant VD change, while others $[16,19,25]$ have documented microvascular improvement after IOP reduction. Zeboulon et al. [20] reported a limited change in the whole peripapillary VD change and increased focal peripapillary vascular loss 1 month after deep nonpenetrating sclerotomy. Lommatzsch et al. [18] reported that no significant changes were detectable in the papillary or macular VD, RNFL, or macular ganglion cell layer thickness after trabeculectomy in open-angle glaucoma patients. Kim et al. [17] showed no significant change in the microcirculation of the peripapillary retina and choroid 3 months postoperatively after trabeculectomy in POAG patients. Contrarily, Shin et al. [19] showed that 19 (61.3\%) of 31 eyes exhibited peripapillary microvascular improvement in the circumpapillary capillary dropout area three months after the trabeculectomy. Hollo et al. [16] also observed pVD improvement after large IOP reduction by topical medication in young patients with high untreated IOP. Liu et al. [25] reported the increase of vessel densities in the optic nerve head and the peripapillary area after applying prostaglandin analog for more than three weeks in the treatment-naïve eyes.

These studies demonstrated peripapillary and macular VD changes after trabeculectomy or large IOP reduction during a relatively short follow-up period (three to six months). However, in this study, we followed up with the patients who underwent trabeculectomy for two years and investigated the correlation between VD change and VF deterioration. To the best of our knowledge, this is the first study describing the association of VD with VF outcome after trabeculectomy. The diagnostic potential of macular and peripapillary OCT-A-determined VD loss in early glaucoma has been reported $[11,15,26]$. Along with these studies, our study showed that the assessment of peripapillary retinal circulation can be used as a predictor of visual function after IOP lowering surgery. GCC thickness change rate and the change rates of other vascular parameters did not show any difference between the deteriorated and the non-deteriorated VF groups and were not related to the postoperative VF MD change rate. Considering that most glaucoma patients who have undergone trabeculectomy already have a substantial loss of VF, a biomarker reflecting visual function change is important in the care of advanced glaucoma patients.

It is unclear why some eyes showed a decrease of peripapillary VD despite successful IOP reduction. A significant IOP decrease after trabeculectomy is known to reduce the depth of the lamina cribrosa [27-29]. Additionally, this level of LC depth reduction has shown an association with microvascular improvement after trabeculectomy [17,19]. However, in another study on the long-term shape and depth of LC after trabeculectomy, although most eyes showed long-term flattening and shallowing of the LC, some eyes showed a deepened LC from the baseline. Therefore, the authors concluded that a reduction of IOP plays an important role in the early phase of LC change; however, LC 
remodeling may play a crucial role in a stable IOP in the later phase [30]. Hence, such remodeling of LC may lead to VD reduction and glaucomatous VF deterioration.

This study has some limitations. First, we excluded 16 eyes due to the poor image quality, poor clarity, and segmentation failure of OCT-A. This could be a limitation of the current OCT-A technology. Second, the study population was of a single ethnicity; thus, the results may not be directly applied to other ethnic groups. Third, the sample size was relatively small, suggesting the need for further study with a larger population. Lastly, the follow-up period was two years postoperatively, which may be relatively short considering that glaucoma is a progressive degenerative disease. A study with a longer duration may confirm our findings.

\section{Conclusions}

This study demonstrated that a greater loss of peripapillary microvasculature after trabeculectomy is associated with visual function deterioration up to two years after surgery. This is the first study showing the relationship between the retinal microvasculature and visual function change after trabeculectomy. POAG patients with greater peripapillary capillary decrease after the IOP lowering surgery may experience VF deterioration; therefore, careful monitoring is warranted in these patients. Additionally, our results suggest the potential use of OCT-A measured peripapillary VD as a biomarker for predicting the visual function after trabeculectomy.

Author Contributions: Conceptualization, J.Y., K.R.S. and J.W.S.; methodology, J.Y., K.R.S. and J.W.S.; formal analysis, J.Y., K.R.S. and J.W.S.; investigation, J.Y., K.R.S. and J.W.S.; resources, J.Y., K.R.S. and J.W.S.; writing—original draft preparation, J.Y.; writing—review and editing, K.R.S. and J.W.S.; supervision, K.R.S. All authors have read and agreed to the published version of the manuscript.

Funding: This research received no external funding.

Institutional Review Board Statement: The study was conducted according to the guidelines of the Declaration of Helsinki, and approved by the Institutional Review Board of Asan Medical Center (IRB no. 2021-1284, date of approval: 19 August 2021).

Informed Consent Statement: Patient consent was waived due to the retrospective nature of this study, and due to the subjects' anonymity and minimal risk to patients.

Data Availability Statement: The data used to support the findings of this study are included in the article, and are available on request from the corresponding author.

Conflicts of Interest: The authors declare no conflict of interest.

\section{References}

1. Weinreb, R.N.; Khaw, P.T. Primary open-angle glaucoma. Lancet 2004, 363, 1711-1720. [CrossRef]

2. Collaborative Normal-Tension Glaucoma Study Group. Comparison of glaucomatous progression between untreated patients with normal-tension glaucoma and patients with therapeutically reduced intraocular pressures. Am. J. Ophthalmol. 1998, 126, 487-497.

3. Heijl, A.; Leske, M.C.; Bengtsson, B.; Hyman, L.; Bengtsson, B.; Hussein, M. Reduction of intraocular pressure and glaucoma progression: Results from the Early Manifest Glaucoma Trial. Arch. Ophthalmol. 2002, 120, 1268-1279. [CrossRef] [PubMed]

4. Gordon, M.O.; Beiser, J.A.; Brandt, J.D.; Heuer, D.K.; Higginbotham, E.J.; Johnson, C.A.; Keltner, J.L.; Miller, J.P.; Parrish, R.K.; Wilson, M.R. The Ocular Hypertension Treatment Study: Baseline factors that predict the onset of primary open-angle glaucoma. Arch. Ophthalmol. 2002, 120, 714-720. [CrossRef]

5. Investigators, A. The Advanced Glaucoma Intervention Study (AGIS): 7. The relationship between control of intraocular pressure and visual field deterioration. Am. J. Ophthalmol. 2000, 130, 429-440.

6. Kass, M.A.; Heuer, D.K.; Higginbotham, E.J.; Johnson, C.A.; Keltner, J.L.; Miller, J.P.; Parrish, R.K.; Wilson, M.R.; Gordon, M.O. The Ocular Hypertension Treatment Study: A randomized trial determines that topical ocular hypotensive medication delays or prevents the onset of primary open-angle glaucoma. Arch. Ophthalmol. 2002, 120, 701-713. [CrossRef]

7. Leske, M.C.; Heijl, A.; Hussein, M.; Bengtsson, B.; Hyman, L.; Komaroff, E. Factors for glaucoma progression and the effect of treatment: The early manifest glaucoma trial. Arch. Ophthalmol. 2003, 121, 48-56. [CrossRef]

8. Yanagi, M.; Kawasaki, R.; Wang, J.J.; Wong, T.Y.; Crowston, J.; Kiuchi, Y. Vascular risk factors in glaucoma: A review. Clin. Exp. Ophthalmol. 2011, 39, 252-258. [CrossRef] 
9. Deokule, S.; Vizzeri, G.; Boehm, A.; Bowd, C.; Weinreb, R.N. Association of visual field severity and parapapillary retinal blood flow in open-angle glaucoma. J. Glaucoma 2010, 19, 293-298. [CrossRef]

10. Logan, J.; Rankin, S.; Jackson, A. Retinal blood flow measurements and neuroretinal rim damage in glaucoma. Br. J. Ophthalmol. 2004, 88, 1049-1054. [CrossRef]

11. Jia, Y.; Wei, E.; Wang, X.; Zhang, X.; Morrison, J.C.; Parikh, M.; Lombardi, L.H.; Gattey, D.M.; Armour, R.L.; Edmunds, B. Optical coherence tomography angiography of optic disc perfusion in glaucoma. Ophthalmology 2014, 121, 1322-1332. [CrossRef]

12. Spaide, R.F.; Klancnik, J.M.; Cooney, M.J. Retinal vascular layers imaged by fluorescein angiography and optical coherence tomography angiography. JAMA Ophthalmol. 2015, 133, 45-50. [CrossRef]

13. Akagi, T.; Iida, Y.; Nakanishi, H.; Terada, N.; Morooka, S.; Yamada, H.; Hasegawa, T.; Yokota, S.; Yoshikawa, M.; Yoshimura, N. Microvascular density in glaucomatous eyes with hemifield visual field defects: An optical coherence tomography angiography study. Am. J. Ophthalmol. 2016, 168, 237-249. [CrossRef]

14. Holló, G. Vessel density calculated from OCT angiography in 3 peripapillary sectors in normal, ocular hypertensive, and glaucoma eyes. Eur. J. Ophthalmol. 2016, 26, e42-e45. [CrossRef]

15. Liu, L.; Jia, Y.; Takusagawa, H.L.; Pechauer, A.D.; Edmunds, B.; Lombardi, L.; Davis, E.; Morrison, J.C.; Huang, D. Optical coherence tomography angiography of the peripapillary retina in glaucoma. JAMA Ophthalmol. 2015, 133, 1045-1052. [CrossRef]

16. Holló, G. Influence of large intraocular pressure reduction on peripapillary OCT vessel density in ocular hypertensive and glaucoma eyes. J. Glaucoma 2017, 26, e7-e10. [CrossRef]

17. Kim, J.-A.; Kim, T.-W.; Lee, E.J.; Girard, M.J.; Mari, J.M. Microvascular changes in peripapillary and optic nerve head tissues after trabeculectomy in primary open-angle glaucoma. Investig. Ophthalmol. Vis. Sci. 2018, 59, 4614-4621. [CrossRef]

18. Lommatzsch, C.; Rothaus, K.; Koch, J.; Heinz, C.; Grisanti, S. Retinal perfusion 6 months after trabeculectomy as measured by optical coherence tomography angiography. Int. Ophthalmol. 2019, 39, 2583-2594. [CrossRef]

19. Shin, J.W.; Sung, K.R.; Uhm, K.B.; Jo, J.; Moon, Y.; Song, M.K.; Song, J.Y. Peripapillary microvascular improvement and lamina cribrosa depth reduction after trabeculectomy in primary open-angle glaucoma. Investig. Ophthalmol. Vis. Sci. 2017, 58, 5993-5999. [CrossRef]

20. Zéboulon, P.; Lévêque, P.-M.; Brasnu, E.; Aragno, V.; Hamard, P.; Baudouin, C.; Labbé, A. Effect of surgical intraocular pressure lowering on peripapillary and macular vessel density in glaucoma patients: An optical coherence tomography angiography study. J. Glaucoma 2017, 26, 466-472. [CrossRef]

21. Verma, S.; Nongpiur, M.E.; Atalay, E.; Wei, X.; Husain, R.; Goh, D.; Perera, S.A.; Aung, T. Visual Field Progression in Patients with Primary Angle-Closure Glaucoma Using Pointwise Linear Regression Analysis. Ophthalmology 2017, 124, 1065-1071. [CrossRef]

22. Ehrnrooth, P.; Puska, P.; Lehto, I.; Laatikainen, L. Progression of visual field defects and visual loss in trabeculectomized eyes. Graefe's Arch. Clin. Exp. Ophthalmol. 2005, 243, 741-747. [CrossRef]

23. Kotecha, A.; Spratt, A.; Bunce, C.; Garway-Heath, D.F.; Khaw, P.T.; Viswanathan, A. Optic disc and visual field changes after trabeculectomy. Investig. Ophthalmol. Vis. Sci. 2009, 50, 4693-4699. [CrossRef]

24. Shigeeda, T.; Tomidokoro, A.; Araie, M.; Koseki, N.; Yamamoto, S. Long-term follow-up of visual field progression after trabeculectomy in progressive normal-tension glaucoma. Ophthalmology 2002, 109, 766-770. [CrossRef]

25. Liu, C.; Umapathi, R.M.; Atalay, E.; Schmetterer, L.; Husain, R.; Boey, P.Y.; Aung, T.; Nongpiur, M.E. The Effect of Medical Lowering of Intraocular Pressure on Peripapillary and Macular Blood Flow as Measured by Optical Coherence Tomography Angiography in Treatment-naive Eyes. J. Glaucoma 2021, 30, 465-472. [CrossRef]

26. Rao, H.L.; Pradhan, Z.S.; Weinreb, R.N.; Reddy, H.B.; Riyazuddin, M.; Dasari, S.; Palakurthy, M.; Puttaiah, N.K.; Rao, D.A.; Webers, C.A. Regional comparisons of optical coherence tomography angiography vessel density in primary open-angle glaucoma. Am. $J$. Ophthalmol. 2016, 171, 75-83. [CrossRef]

27. Lee, E.J.; Kim, T.-W. Lamina cribrosa reversal after trabeculectomy and the rate of progressive retinal nerve fiber layer thinning. Ophthalmology 2015, 122, 2234-2242. [CrossRef]

28. Lee, E.J.; Kim, T.-W.; Weinreb, R.N.; Kim, H. Reversal of lamina cribrosa displacement after intraocular pressure reduction in open-angle glaucoma. Ophthalmology 2013, 120, 553-559. [CrossRef]

29. Quigley, H.; Arora, K.; Idrees, S.; Solano, F.; Bedrood, S.; Lee, C.; Jefferys, J.; Nguyen, T.D. Biomechanical responses of lamina cribrosa to intraocular pressure change assessed by optical coherence tomography in glaucoma eyes. Investig. Ophthalmol. Vis. Sci. 2017, 58, 2566-2577. [CrossRef]

30. Kadziauskienè, A.; Jašinskienè, E.; Ašoklis, R.; Lesinskas, E.; Rekašius, T.; Chua, J.; Cheng, C.-Y.; Mari, J.M.; Girard, M.J.; Schmetterer, L. Long-term shape, curvature, and depth changes of the lamina cribrosa after trabeculectomy. Ophthalmology 2018, 125, 1729-1740. [CrossRef] 